\title{
Status and mating success amongst visual artists
}

\author{
Helen Clegg ${ }^{1}{ }^{*}$, Daniel Nettle ${ }^{2}$ and Dorothy Miell ${ }^{3}$ \\ 1 Division of Psychology, The University of Northampton, Northampton, UK \\ ${ }^{2}$ Centre for Behaviour and Evolution, Institute of Neuroscience, Newcastle University, Newcastle, UK \\ ${ }^{3}$ College of Humanities and Social Sciences, University of Edinburgh, Edinburgh, UK
}

Edited by:

Simine Vazire, Washington University in St. Louis, USA

Reviewed by:

Geoffrey Miller, University of New

Mexico, USA

Elisabeth Oberzaucher, University of

Vienna, Austria

\section{${ }^{*}$ Correspondence:}

Helen Clegg, Division of Psychology,

The University of Northampton, Park

Campus, Boughton Green Road,

Northampton NN2 7AL, UK.

e-mail: helen.clegg@northampton.

ac.uk
Geoffrey Miller has hypothesized that producing artwork functions as a mating display. Here we investigate the relationship between mating success and artistic success in a sample of 236 visual artists. Initially, we derived a measure of artistic success that covered a broad range of artistic behaviors and beliefs. As predicted by Miller's evolutionary theory, more successful male artists had more sexual partners than less successful artists but this did not hold for female artists. Also, male artists with greater artistic success had a mating strategy based on longer term relationships. Overall the results provide partial support for the sexual selection hypothesis for the function of visual art.

Keywords: artist, mating success, status, evolutionary psychology, creativity

\section{INTRODUCTION}

Various creative behaviors, including art, do not fit the adaptationist criteria for survival mechanisms, which include low phenotypic and genotypic variance, low heritability, and universality across individuals (Miller, 2000). Therefore, Miller (2000) proposes that these behaviors may be products of mechanisms that evolved through sexual selection. According to this theory, artworks should act as indicators of genetic and phenotypic quality, whose function is to attract mates, and so it follows that successfully creative individuals should have greater mating success.

There is some evidence from mate preference studies that creativity is desired in a potential mate. In a cross-cultural study on mate preferences overall males and females ranked "creative and artistic" as 7th and 6th respectively out of 13 traits and there was little effect of culture on preferences for these characteristics (Buss et al., 1990). Also, in a study considering the trade-offs people make in mate preferences, once men had found a partner with evidence of enough physical attractiveness and women had found a partner with enough resources then creativity was the characteristic most likely to be sought by both men and women (Li et al., 2002). However, in terms of artistic production Miller (1999) claimed that there will be sexual dimorphism despite evidence for mutual mate choice in this trait. Support for this has been found in studies that demonstrated that male output for creative products, such as artwork, books, and scientific discoveries, was approximately 10 times that of females (Miller, 1999; Kanazawa, 2000). Nevertheless, strong cultural and social expectations of gender roles and stereotypes may play an important part in these behaviors.

There is also evidence that creativity is enhanced when individuals are primed with thoughts or images of potential mates (Griskevicius et al., 2006). Interestingly, Griskevicius et al. (2006) found a sex difference in terms of the type of prime required to increase creativity. Men were more creative compared to controls when primed with a short- or long-term mate whereas this effect was only found for women when they were primed with a scenario regarding a high-quality, committed long-term mate. It is likely however that variation in the menstrual cycle may influence desire for creative individuals. In fact, it has been found that in naturally cycling women, current fertility is positively correlated with preference for creativity in a short-term mate (Haselton and Miller, 2006), suggesting that creative output acts as an indicator of "good genes." These findings are consistent with parental investment and sexual selection theory.

It is only recently that empirical research has been conducted to test the prediction of increased mating success for highly creative individuals. A study by Nettle and Clegg (2006) found that both professional and serious artists had significantly more sexual partners than a control group. However, their artistic success was self-perceived, a somewhat subjective measure. Moreover, the Nettle and Clegg (2006) study compared two groups who have differed in a wide variety of lifestyle factors that could have produced the result. Therefore, in this study we test a more subtle prediction of Miller's theory, namely that amongst people who all produce visual art, those who are more successful at doing so should have greater mating success, than those who are less successful.

We made three predictions. Firstly, in line with Miller's hypothesis that creativity is a sexually selected trait, more successful artists will have significantly increased mating success compared to less successful artists. Secondly, because of strategic differences between the sexes and consistent with evidence of sexual dimorphism in creative production, this effect will be observed in male artists but not female artists. Thirdly, since sexual selection theory predicts that those with good quality fitness indicators will have the opportunity to engage in an increased number of sexual liaisons with different mates we predicted that more successful male artists will have a more short-term mating strategy compared to less successful male artists. 


\section{MATERIALS AND METHODS}

Data were collected from self-identified visual artists using an online questionnaire. The first author worked closely with two artists in the construction of a set of questions that assayed success within this community. Artists were initially asked whether they considered themselves to be professional, serious, or hobby artists and this variable acted as a measure of self-perceived artistic success. The other questions in this section examined more diverse measures of artistic success. These were how long they had been an artist, time spent on art per week, number of exhibitions in past 5 years, average length of time of an exhibition, minimum and maximum cost of a piece of artwork, percentage of income made from artwork over the past 12 months, importance of art in the person's life, the degree to which they believe that art is about taking risks, the importance of being recognized by the general public, and the importance of being recognized by other artists as an artist (the last four questions were rated on a scale from 1 to 10$)$.

The final section consisted of demographic and sexual behavior questions. Numbers of biological children were requested as this acts as a measure of achieved fertility (Perusse, 1993; Hopcroft, 2004). However, it should be noted that men tend to, if anything, undercount their offspring and so it is possible that the findings may underestimate male artists' achieved fertility (Hopcroft, 2004). Lifetime number of sexual partners was asked as an indicator of the ability to attract mates and is a commonly used measure of mating success (Faurie et al., 2004; Nettle and Clegg, 2006). The participants were also asked about the length of the relationships they had had as an indicator of mating strategy. Participants were provided with eight options ranging from "one night stands" to "lasting over 10 years" for which they put the number of relationships they had experienced for each relationship length.

The sample was primarily recruited from an advert in two artists' magazines, one of which predominantly caters for professional artists and one which tends to attract more amateur/hobby artists. Information about the study was also posted on the websites of a number of artists' groups, both professional and amateur, which have a presence on the internet. Amateur/hobby artists were also recruited by advertising on the Intranet at a University in the South of England and another site which publicizes online psychological research.

\section{RESULTS}

The sample consisted of 236 heterosexual artists of whom 85 (36\%) were men and 151 (64\%) were women. The age range was from 18 to 78 years, with a mean of 42.67 years and SD of 13.22, and approximated a normal distribution. Ninety-one percent of participants were from a white western background.

\section{ARTISTIC SUCCESS}

To develop a broadly based measure of artistic success, we conducted an unrotated principal components analysis (PCA) on the variables concerning artistic success. Any missing values were replaced with the mean. After an initial PCA was conducted the variable "Art is about taking risks" was removed due to low communality and a second PCA performed. A three-factor solution accounted for $63 \%$ of the variance.
All variables loaded on factor one, from 0.246 to 0.802 , with the majority loading above 0.400 . The variables were (with loadings on factor 1 in brackets) "self-reported artistic success" (0.802), "time spent on art" (0.743), "public display" (number of days artists have displayed their work over the past 5 years; 0.246$)$, "minimum cost of art" (0.433), "maximum cost of art" (0.399), "percentage of income from art" (0.677), "importance of art in life" (0.698), "importance of public recognition" (0.506), and "importance of recognition from other artists" (0.515). Factor one accounted for $34 \%$ of the total variance. Therefore this factor will be taken as the measure of artistic status.

\section{MATING SUCCESS}

The distribution of number of sexual partners for these participants was highly skewed with a minimum of 0 and a maximum of $250(M=10.67, \mathrm{SD}=21.75)$. Therefore, the data were converted to a log scale and the following statistics computed using this scale.

A multiple regression was conducted with mating success as the dependent variable and artistic success, age, income, and length of current relationship as the independent variables. Separate multiple regressions were conducted for males and females as it is predicted by evolutionary theory that different variables will predict mating success for the two sexes. For males, the model was significant $[F(4,50)=8.387, p=0.001]$ and accounted for $35 \%$ of the variance (adjusted $R^{2}=0.354$ ). Artistic status was the only predictor variable that reached significance $(\beta=0.546, p=0.001)$. Bivariate correlations of the variables that make up the artistic success variable with male artists' mating success showed that "time spent on art" $(r=0.592, p=0.001)$, "self-reported status" $(r=0.470, p=0.001)$, "percentage of income from art" $(r=0.255$, $p=0.048)$, "importance of art in life" $(r=0.361, p=0.004)$, "importance of public recognition" $(r=0.280, p=0.027)$, “importance of recognition from other artists" $(r=0.340, p=0.007)$ were all significantly correlated with mating success.

When a multiple regression analysis was conducted for female artists, using the same variables as for males, the model was significant $[F(4,95)=5.734, p=0.001]$ and accounted for $16 \%$ of the variance (adjusted $R^{2}=0.161$ ). Length of relationship was the strongest predictor variable of female artists' mating success $(\beta=-0.560, p=0.001)$ with age of artist also being a significant predictor $(\beta=0.296, p=0.029)$. None of the other variables, including artistic success were significant predictors for female artists' mating success.

\section{MATING STRATEGY}

To measure differences in mating strategy, a mating strategy index was computed in the following way. Each one night stand gained one point, each relationship up to a month two points, and so on up to each relationship 10 years or over which gained eight points. The total number of points for each person was added up and divided by their total number of relationships. Thus, the lower their score the more short-term their mating strategy. Since the distribution was highly skewed the data were converted to a log scale and the following statistics carried out using this scale.

A multiple regression was conducted separately for males and females, with mating strategy as the dependent variable, and 
artistic success, age, length of current relationship, and income over the last 12 months as the independent variables. Ideally, whether the artists had children or not should also have been included as mating strategy has been shown to change once the individual has offspring. However the available data on children had a large number of missing values and it was not clear whether these individuals did not have children or whether they had chosen not to respond to these questions. Therefore numbers of offspring were not included in the regression.

For males, the model was significant $[F(4,32)=8.993$, $p=0.001]$ and accounted for $47 \%$ of the variance (adjusted $\left.R^{2}=0.470\right)$. Artistic status was the only significant predictor variable $(\beta=0.579, p=0.001)$. Bivariate correlations of the variables that make up the artistic success variable with male artists' mating strategy demonstrated that "time spent on art" $(r=0.528$, $p=0.001)$, "self-reported artistic status" $(r=0.507, p=0.001)$, "minimum cost of art" $(r=0.319, p=0.047)$, and "percentage of income from art" ( $r=0.446, p=0.004)$ were all significantly correlated with male mating strategy.

For females the model was significant $[F(4,74)=3.209$, $p=0.017]$ and accounted for $10 \%$ of the variance (adjusted $\left.R^{2}=0.102\right)$. Length of current relationship was the strongest significant predictor variable $(\beta=-0.536, p=0.001)$. Age was also a significant predictor of mating strategy $(\beta=0.453, p=0.005)$. None of the other variables were significant including artistic success.

\section{DISCUSSION}

Although, according to the sexual selection hypothesis of creativity, it is actually quality of art that attracts sexual partners this is a subjective concept that is difficult to measure. The alternative tactic followed here is to consider other aspects of artistic behavior that may suggest professional success. Whilst we recognize that since the participants were asked to supply the information that made up the integrated success variable it remains a self-report measure, it is likely to reflect objective success in the artistic domain reasonably well. Although we acknowledge that this is not a truly integrated or comprehensive measure of artistic success, it is arguable whether any quantitative measure could truly capture this and it is certainly an improvement on previous self-reported measures of artistic success. Considering the variables that made up the artistic success variable, public display, minimum and maximum cost of art, and percentage of income earned from art could be taken as indicators of public endorsement of the artist's work and as such could be argued to be indicators of quality of the artwork as perceived by the community. Time spent on artwork is indicative of the role art plays in the artist's life and can be considered as an indicator of quality of the artwork since according to the equal-odds rule (Simonton, 1988, 1997) the more work a creative individual produces the more pieces of work of high-quality the individual is likely to produce. Moreover it takes a creative individual approximately 10 years in their chosen field before they can build up the necessary knowledge and skill to produce a product that is truly innovative (Simonton, 1999). Hence, length of time as an artist is also likely to be closely associated with quality of artwork. Importance of art in life, recognition from the general public and recognition from other artists are also relevant to artistic success.
Bain (2005) highlights the difficulties for artists of being recognized as such, due to lack of requirement of formal qualifications and no shared workplace. Therefore recognition from others of their identity as artists as well as an importance of art in their lives constructs a narrative that projects an acknowledgment of their professional status (Bain, 2005). Thus, the measure of artistic status within this study is a broad based measure of artistic status that captures a comprehensive variety of behaviors and beliefs held by artists.

The results for mating success and artistic success are consistent with the sexual selection hypothesis of creativity, with more professional male artists having more sexual partners than less professional male artists but this relationship is not found for female artists. This is the case for male artists even when income, a commonly cited desirable trait in potential partners for women, is included. These results are supported by a study by Haselton and Miller (2006) that found that women in their most fertile period of their menstrual cycle preferred poor but creative men over wealthy, non-creative individuals as short-term mates. Furthermore, although significant, the model only accounts for $35 \%$ of the variance for males. Further research is required to consider other relevant variables to mating success and how these may interact with artistic success to effect numbers of sexual partners gained.

One possible interpretation of these results is that what is attracting more partners is high status rather than creativity per se. However, it is not always clear what is meant by the term status. If it is accumulation of resources such as income then although income was found to be significantly correlated with mating success, once this was controlled for objective artistic success remained a significant predictor of numbers of sexual partners for male artists. However, if the term status is used as an indicator of how successful one is in one's chosen field then, within the particular community we have studied, this is inseparable from creative acclaim.

The non-significant finding for female artists may not mean that professional female artists are not attracting sexual partners through their creativity, but rather that they are seeking quality over quantity. This can not be confirmed or refuted from the data available in this study. However, Griskevicius et al. (2006) found that women's creativity increased when primed with a stimulus for a long-term, committed, high-quality mate but not when the primes for a short-term, potential long-term or control were presented. This suggests that more professional female artists may be channeling their creativity toward attracting mates of high-quality rather than increasing numbers of mates. Further research using a sample of female artist is required before such conclusions can be drawn.

Contrary to prediction, more successful male artists were found to employ longer term mating strategies. This appears to be at odds with the finding that more professional artists had greater numbers of sexual partners. It may be that more successful artists have a greater choice of potential partners, and males therefore sample a greater number before choosing one to have a longer term relationship with which then endures. Another possibility is that more successful artists are more likely to have extra pair copulations whilst in a long-term relationship. Further research is required to understand the mating strategies of artists of differing 
professional success. This should include a consideration of presence or absence of offspring, which has found to influence mating strategy in previous studies.

\section{CONCLUSION}

The findings reported here are generally consistent with the sexual selection hypothesis for the maintenance of artistic behaviors.

\section{REFERENCES}

Bain, A. (2005). Constructing an artistic identity. Work Employ. Soc. 19, 25-46.

Buss, D. M., Abbott, M., Angleitner, A., Asherian, A., Biaggio, A., BlancoVillasenor, A., Bruchon-Schweitzer, M., Ch'U, H. Y., Czapinski, J., Deraad, B., Ekehammar, B., El Lohamy, N., Fioravanti, M., Georgas, J., Gjerde, P., Guttman, R., Hazan, F., Iwawaki, S., Janakiramaiah, N., Khosroshani, F., Kreitler, S., Lachenicht, L., Lee, M., Liik, K., Little, B., Mika, S., Moadel-Shahid, M., Moane, G., Montero, M., MundyCastle, A. C., Niit, T., Nsenduluka, E., Pienkowski, R., Pirtila-Backman, A.-M., De Leon, J. P., Rousseau, J., Runco, M. A., Safir, M. P., Samuels, C., Sanitioso, R., Serpell, R., Smid, N., Spencer, C., Tadinac, M., Todorova, E. N., Troland, K., Van Den Brande, L., Van Heck, G., Langenhove, L. V., and Yang, K.-S. (1990). International preferences in selecting mates. A study of 37 cultures. J. Cross Cult. Psychol. 21, 5-47.

Faurie, C., Pontier, D., and Raymond, M. (2004). Student athletes claim to have more sexual partners than other students. Evol. Hum. Behav. 25, $1-8$.

Griskevicius, V., Cialdini, R. B., and Kenrick, D. T. (2006). Peacocks, Picasso, and parental investment: the effects of romantic motives on creativity. J. Pers. Soc. Psychol. 91, 63-76.

Haselton, M., and Miller, G. F. (2006). Women's fertility across the cycle increases the short-term attractiveness of creative intelligence compared to wealth. Hum. Nat. 17, 50-73.

Hopcroft, R. L. (2004). Those who can do, except those who think about it: sex, status and reproductive success in the contemporary US. Paper Presented at the Human Behaviour and Evolution Society, Berlin.

Kanazawa, S. (2000). Scientific discoveries as cultural displays; a further test of Miller's courtship model. Evol. Hum. Behav. 21, 317-321.

Li, N. P., Bailey, J. M., Kenrick, D. T., and Linsenmeier, J. A. W. (2002). The necessities and luxuries of mate preferences: testing the tradeoffs. J. Pers. Soc. Psychol. 82, 947-955.

Miller, G. F. (1999). "Sexual selection for cultural displays," in The

This study has considered a group of artists who may vary on a number of different personality, lifestyle, and socioeconomic measures. However, despite this variation, there was a significant association between artistic success and mating success. Further research is needed to explore whether these findings cut across cultures and sub-cultures, and different types of creative behavior.

Evolution of Culture, eds R. Dunbar, C. Knight, and C. Power (Edinburgh: Edinburgh University Press), 71-91.

Miller, G. F. (2000). "Mental traits as fitness indicators: expanding evolutionary psychology's adaptationism," in Evolutionary Approaches to Human reproductive Behavior, Vol. 709, eds D. LeCroy and P. Moller (New York, NY: Annals of the New York Academy of Science), 62-74.

Nettle, D., and Clegg, H. (2006). Schizotypy, creativity and mating success in humans. Proc. R. Soc. Lond. B Biol. Sci. 273, 611-615.

Perusse, D. (1993). Cultural and reproductive success in industrial societies: testing the relationship at the proximate and ultimate levels. Behav. Brain Sci. 16, 267-322.

Simonton, D. K. (1988). Age and outstanding achievement: what do we know after a century of research? Psychol. Bull. 104 251-267.

Simonton, D. K. (1997). Creative productivity: a predictive and explanatory model of career trajectories and landmarks. Psychol. Rev. 104, 66-89.
Simonton, D. K. (1999). Origins of Genius. Darwinian Perspectives on Creativity. New York: Oxford University Press.

Conflict of Interest Statement: The authors declare that the research was conducted in the absence of any commercial or financial relationships that could be construed as a potential conflict of interest.

Received: 19 July 2011; paper pending published: 26 July 2011; accepted: 14 October 2011; published online: 31 October 2011.

Citation: Clegg H, Nettle D and Miell $D$ (2011) Status and mating success amongst visual artists. Front. Psychology 2:310. doi: 10.3389/fpsyg.2011.00310

This article was submitted to Frontiers in Personality Science and Individual Differences, a specialty of Frontiers in Psychology.

Copyright (c) 2011 Clegg, Nettle and Miell. This is an open-access article subject to a non-exclusive license between the authors and Frontiers Media SA, which permits use, distribution and reproduction in other forums, provided the original authors and source are credited and other Frontiers conditions are complied with. 